\title{
Training for tutors, an innovation in the traditional Brazilian Young Leaders in Diabetes Training
}

Mark Thomaz Ugliara Barone ${ }^{1}$, Lucas Xavier $^{1.2}$, Matheus Chaluppe ${ }^{1}$, Bruna Talita Patricio ${ }^{1}$ Pedro Ripoli ${ }^{1.2}$, Lucas Leme Galastri ${ }^{1}$, Ronaldo Jose Pineda-Wieselberg ${ }^{1.2 .3}$

1 - Brazilian Young Leaders in Diabetes Training, ADJ Diabetes Brasil, SP, Brazil.

2 - Young Leaders in Diabetes Programme, International Diabetes Federation (IDF), Brussels, Belgium. 3 - Faculdade de Ciências Médicas da Santa Casa de Misericórdia de São Paulo, SP, Brazil.
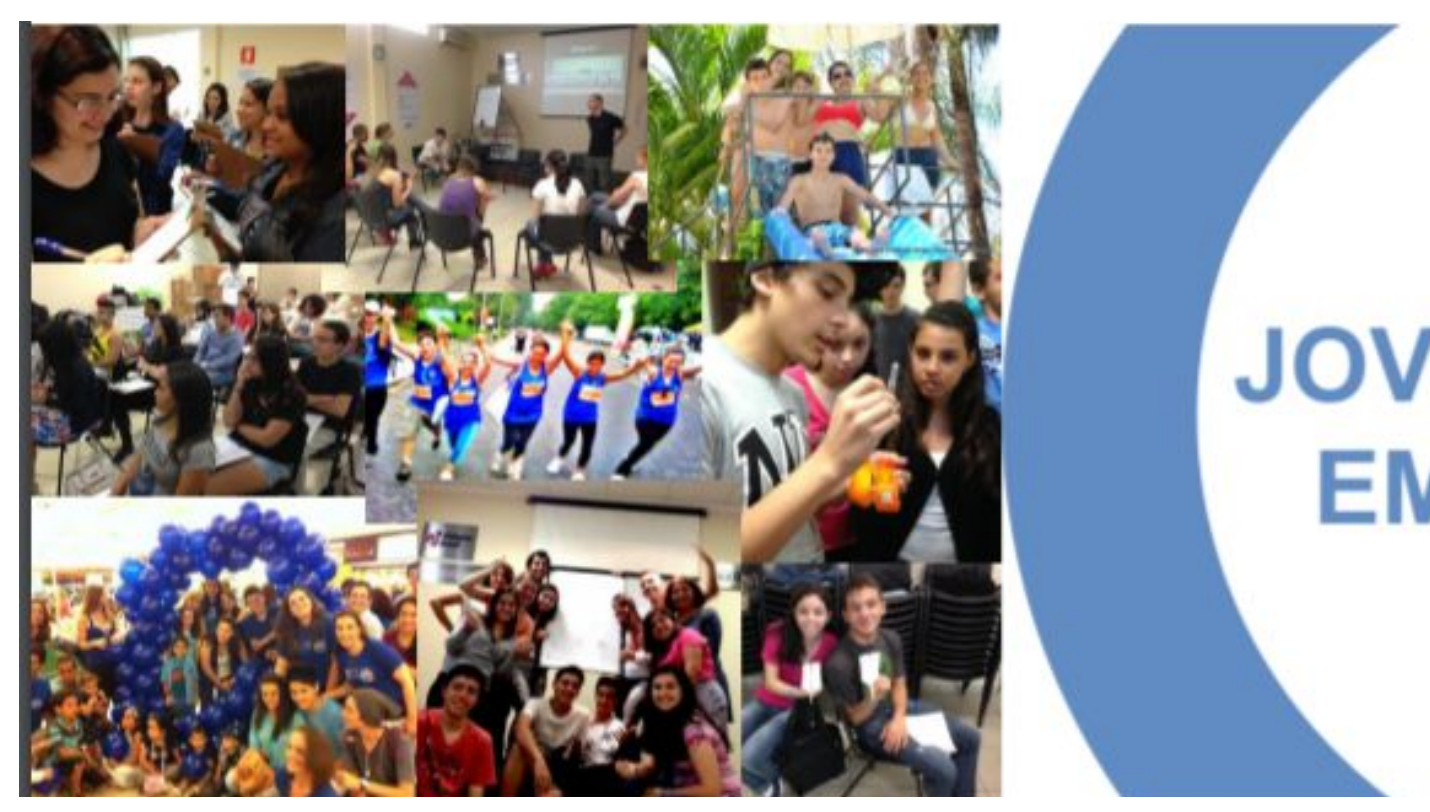

Background

Since 2009, ADJ Diabetes Brasil has been offering the Young Leaders in Diabetes (YLD) Training, a semester-long training for individuals between 15 and 25 years old. In addition to the theoretical and hands-on sessions, the YLD develop new or engage in traditional projects at ADJ Diabetes Brasil, aiming to improve diabetes education and engagement, and leading to increased access to care and life quality. Individuals who stand out during the training are invited to take an additional training to become tutors of the following year YLD Training. The training for tutors is comprised of 91 hours of theory and 21 of practical activities, through workshops, online courses, presentations and mediated peer discussions. It focuses mainly on useful skills for assisting the new YLD to successfully become leaders, completing the training successfully. It includes: didactics, negotiation, persuasion, trust-building, communication, motivation, group dynamics conduction, resilience and diabetes education. Moreover, tutors are requested to take one or more of the online courses on the United Patients Academy website to increase their capacity to use and teach advocacy, fundraising, strategic planning, volunteer management, and targeted communication.

\section{Aims}

Evaluate tutors' knowledge in comparison to new YLD and identify the components of the training for tutors that were the most relevant.

\section{Method}

A test composed of 35 questions - 23 from the Michigan Diabetes Knowledge Test (DKT), 9 multiple-choice and 3 open-ended questions based on IDF guidelines - was taken by 5 tutors (before starting the training for tutors; median age $20 y \pm 0.7)$ and, eight months later, by 6 new candidates to YLD (who, after 6 months, completed the training; median age $20 y \pm 1.2)$. A Mann Whitney U-test was performed to compare grades. Each tutor was asked about the two main skills that he/she developed during the training for tutors that were the most useful to assist candidates to YLD.
The authors declare that they have no conflict of interest with the present poster. This poster does not contain any trade names. This poster does not cover any unapproved uses of specific drugs, other products or devices.

\section{Results}

The difference between grades obtained by the YLD and the tutors was significant $(6.0 \pm 1.0$ vs. $9.1 \pm 0.1 ; p<0.007)$. The most useful skills, according to the tutors, were grouped into two categories (in parenthesis, the number of times that each skill was mentioned):

1) negotiation(2)/persuasion(1)/motivation(1);

2) communication(3)/trust-building(2).

Two other skills cited were: resilience(1) and group management(1).

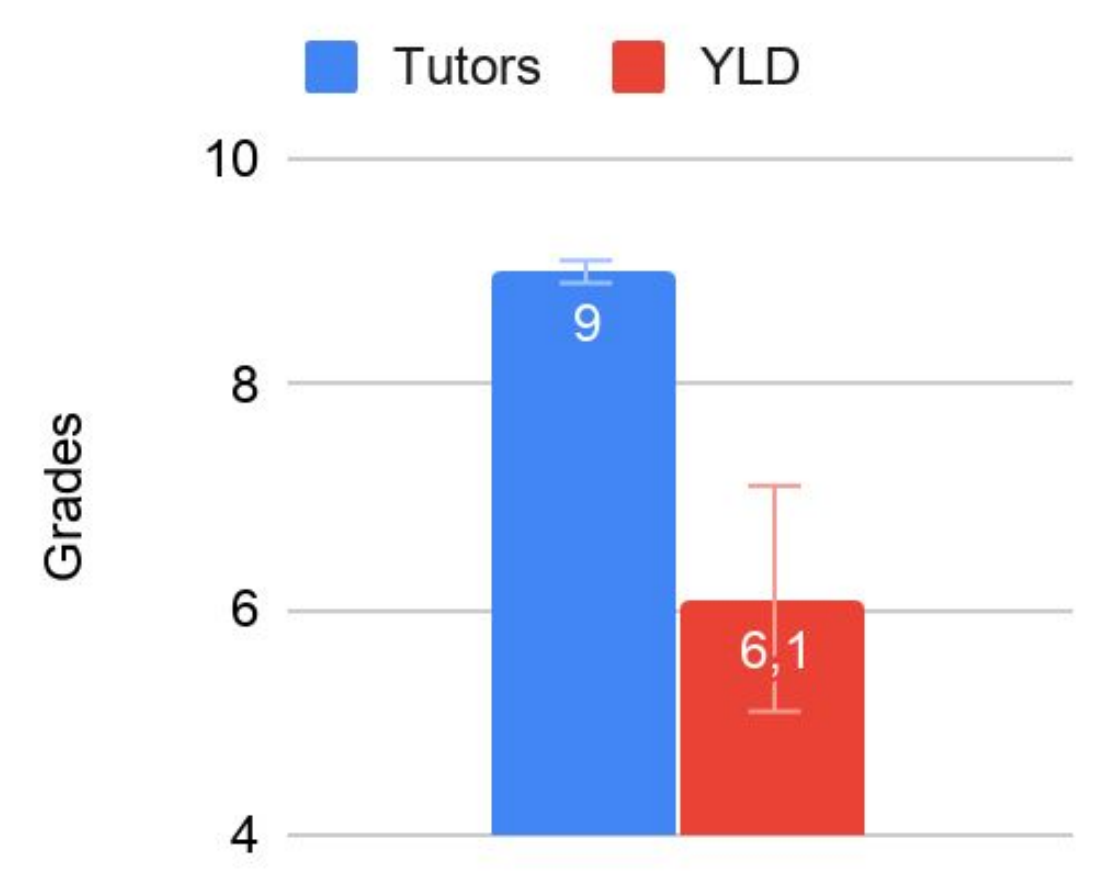

\section{Discussion}

Despite having more knowledge than YLD candidates, since they had completed the YLD Training, Tutors benefited taking the extra training that built valuable capabilities, providing them with tools and confidence to perform their role as tutors to new YLD. We interpret from the tutors' answers that interpersonal skills are the ones most useful to be developed. Interestingly, some of those tutors also reached the highest steps on the empowerment ladder, having overcome the self-acceptance phase, being engaged in the community, or even a higher level of societal influence. Thus, possibly the training for tutors contributes to climb the empowerment ladder (to be further studied).

\section{EMPOWERMENT LADDER}

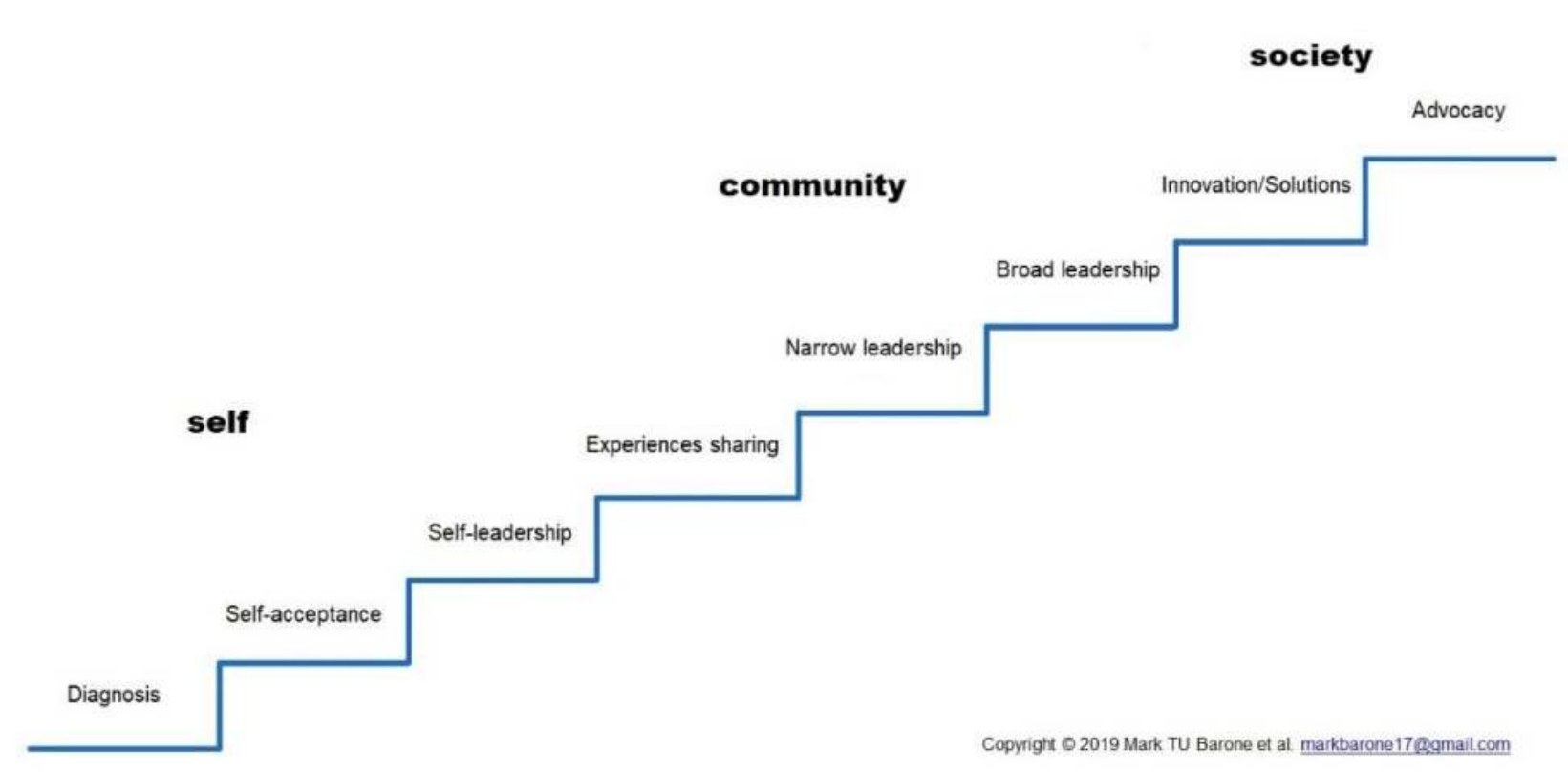

